\title{
Intravitreal Ranibizumab Injection for the Treatment of Retinopathy of Prematurity
}

\author{
Tariq Aldebasi ${ }^{a}$ b Muataz A. Guma ${ }^{a}$ Rabia Bashir ${ }^{a}$ Saif Al Saif ${ }^{c}$ \\ Waleed A. Altwaijrib, c Abdulkareem M. Al Bekairy ${ }^{d}$ \\ a Division of Ophthalmology, Department of Surgery, King Abdul Aziz Medical City, Riyadh, Saudi Arabia; \\ ${ }^{b}$ College of Medicine, King Saud bin Abdulaziz University for Health Sciences, National Guard Health Affairs, \\ Riyadh, Saudi Arabia; 'Neonatal Intensive Care Department, King Abdul Aziz Medical City, Riyadh, Saudi Arabia; \\ ${ }^{\mathrm{d} C o l l e g e ~ o f ~ P h a r m a c y, ~ K i n g ~ S a u d ~ b i n ~ A b d u l a z i z ~ U n i v e r s i t y ~ f o r ~ H e a l t h ~ S c i e n c e s, ~ N a t i o n a l ~ G u a r d ~ H e a l t h ~ A f f a i r s, ~}$ \\ Riyadh, Saudi Arabia
}

\section{Significance of the Study}

- This study contributes useful information on the treatment of retinopathy of prematurity, a leading cause of childhood blindness in the world. This study provides evidence for the successful use of a single dose of $0.3 \mathrm{mg}$ intravitreal ranbizumab in treating advanced stage III retinopathy.

\section{Keywords}

Anti-VEGF · Intravitreal injection · Retinopathy of prematurity · Ranibizumab · Childhood blindness . Vitreoretinal fibrosis

\footnotetext{
Abstract

Objectives: To evaluate the efficacy of a single injection of $0.3 \mathrm{mg}$ intravitreal ranibizumab for the treatment of retinopathy of prematurity (ROP). Methods: We conducted this retrospective case series study at King Abdul Aziz Medical City, Riyadh, Saudi Arabia. Seventy-four eyes of 37 preterm infants with ROP stage III with plus disease in zone I, posterior zone II, and aggressive posterior $\mathrm{ROP}$ received a single injection of $0.3 \mathrm{mg}$ intravitreal ranibizumab. The favorable out-
}

come measure was complete regression of the disease with normal vascularization of the retina of those infants. Results: The gestational age of the 37 included cases was in the range of 23-28 weeks and their body weight at birth was between 510 and 1,235 g except for one case with 2,550 g under oxygen therapy <7days with severe hypoglycemia. All eyes showed a favorable response in terms of regression of plus disease from the first day after treatment, followed by regression of stage III retinopathy. All patients developed complete vascularization over variable periods of time. Conclusion: One injection of $0.3 \mathrm{mg}$ intravitreal ranibizumab is effective in treating ROP stage III mainly in zones I and II.

(C) 2019 The Author(s)

Published by S. Karger AG, Basel

\begin{tabular}{ll}
\hline KARGER & $\begin{array}{l}\text { @ } 2019 \text { The Author(s) } \\
\text { Published by S. Karger AG, Basel }\end{array}$ \\
$\begin{array}{l}\text { Openger } \\
\text { E-Mail karger@karger.com }\end{array}$ & $\begin{array}{l}\text { This is an Open Access article licensed under the Creative Commons } \\
\text { Attribution-NonCommercial-4.0 International License (CC BY-NC) } \\
\text { www.karger.com/mpp }\end{array}$ \\
$\begin{array}{l}\text { (http://www.karger.com/Services/OpenAccessLicense), applicable to } \\
\text { the online version of the article only. Usage and distribution for com- } \\
\text { mercial purposes requires written permission. }\end{array}$
\end{tabular}

Abdulkareem M. Al Bekairy

College of Pharmacy, King Saud bin Abdulaziz University for Health Sciences National Guard Health Affairs, Mail Code 3160, PO Box 3660

Riyadh 11426 (Saudi Arabia)

E-Mail Bekairya@ngha.med.sa 


\section{Introduction}

Retinopathy of prematurity (ROP) is a proliferative vascular disorder of the retina affecting premature infants. It is characterized by aberrant retinal vascularization, vitreoretinal fibrosis, macular dragging, and eventually retinal detachment with grave visual outcome [1]. Several studies have shown that the lower the gestational age and birth weight, the higher the chance of developing ROP [2]. Premature infants with associated neonatal morbidities like respiratory distress syndrome, bronchopulmonary dysplasia, sepsis, intraventricular hemorrhage, poor weight gain, and hyperglycemia are at greatest risk of developing this disease [3]. As the survival of very immature infants has improved with advances in neonatal care, the incidence of ROP is also rising. ROP has become the leading cause of preventable childhood blindness in the world [4]. However, timely diagnosis and treatment can prevent serious visual outcomes.

ROP was first recognized as an important cause of blindness in high-income countries in the 1940s and 50s when survival of very-low-birth-weight babies $(<1,500 \mathrm{~g}$ or approximately $<32$ weeks' gestational age) was improving in association with the widespread use of unrestricted oxygen supplementation. This was termed as the first epidemic of ROP which was caused by oxygen supplementation [5]. Restricting the use of oxygen has reduced the incidence of ROP, but it has increased mortality and the risk of cerebral palsy. Since the 1970s, survival of very-lowgestational-age babies (i.e., $<28$ weeks) has improved in high-income countries as a result of better neonatal care. This was associated with ROP and is recognized as the second epidemic of ROP. ROP is now an increasingly important cause of preventable blindness in China, Southeast Asia, Latin America, and parts of Eastern Europe [6]. In the United States, of 3.9 million infants born each year, about 14,000 are affected by ROP. Among them, 1,100$1,500(7.5-10.7 \%)$ develop disease severe enough to need treatment, and 400-600 infants (2.8-4.2\%) become legally blind from ROP each year [7]. This incidence has risen over the years, as it was reported in 2008 to be only $0.12 \%$ [8]. Eighty-six percent of the premature infants born in the UK with birth weight $<1,500 \mathrm{~g}$ survive, and the incidence of stage III ROP is about 8-10\% [9].

Vascular endothelial growth factor (VEGF) plays an important role in the pathogenesis of ROP. This growth factor is expressed from immature avascular retina, and its levels are regulated by relative oxygen saturation of the tissue. Accumulation of VEGF leads to anomalous neovascularization with its subsequent effects [10]. Elevated
VEGF levels have been detected in patients with ROP [11]. Thus, one approach to treating ROP is aimed at destroying VEGF-producing cells. Laser therapy has largely replaced cryotherapy over the past decades. Both therapies destroy the peripheral retina leading to loss of peripheral vision with high risk of tunnel visual field [12].

More recently, intravitreal injection of anti-VEGF agents, especially bevacizumab, has emerged as an alternative treatment in an effort to salvage peripheral vision. Anti-VEGF therapy is reported to allow normal vascularization of peripheral retina [13]. The better refractive outcomes and the avoidance of the risks of general anesthesia are considered significant advantages of anti-VEGF treatment $[13,14]$. Ranibizumab has been introduced as an alternative to bevacizumab with a number of advantages including lack of penetration of the retina, short half-life, and accordingly much less systemic toxicity potential, and higher general safety profile [15].

Among 9,500 babies born in 2016 in King Abdulaziz Medical City (KAMC), Riyadh, Saudi Arabia, 940 were preterm, comprising almost $10 \%$. Among them, 833 were screened for ROP, and 16 of them (1.7\%) were diagnosed with advanced ROP. This means that almost $2 \%$ of all premature babies with ROP might need treatment every year. This high incidence of ROP is mainly due to the development in neonatal healthcare.

This study was aimed at evaluating the efficiency of using $0.3 \mathrm{mg}$ ranibizumab, an off-label treatment, via intravitreal injection in 74 eyes of 37 preterm infants followed up from July 2012 to December 2016.

\section{Materials and Methods}

This case series study was conducted in the Neonatal Intensive Care Unit and Intermediate Care Unit at KAMC, Riyadh, Saudi Arabia. The study is a joint effort of the Division of Ophthalmology, in collaboration with the Neonatology Department (KAMC), and the College of Pharmacy at King Saud Bin Abdulaziz University for Health Sciences.

This study was approved by the Institutional Review Board of King Abdullah International Medical Research Center, and both verbal and written informed consents were obtained from the parents of all patients. Detailed information on single-dose ranibizumab was provided to the parents. The pros and cons of the old approaches of treatment using cryotherapy and laser were also explained to the parents. The advantages and risks associated with this intravitreal anti-VEGF (ranibizumab) were also explained to all parents.

The study population included all preterm infants admitted to the Neonatal Intensive Care Unit and Intermediate Care Unit from July 2012 to December 2016 with a gestational age $\leq 31$ weeks at birth and a birth weight $\leq 1,500 \mathrm{~g}$. Infants with a gestational age 
Table 1. Schedule for the timing of initial screening and follow-up examinations

\begin{tabular}{|c|c|c|c|c|}
\hline Timing & 1 week or less & 1-2 weeks & 2 weeks & 2-3 weeks \\
\hline $\begin{array}{l}\text { ROP stage } \\
\text { and zone }\end{array}$ & 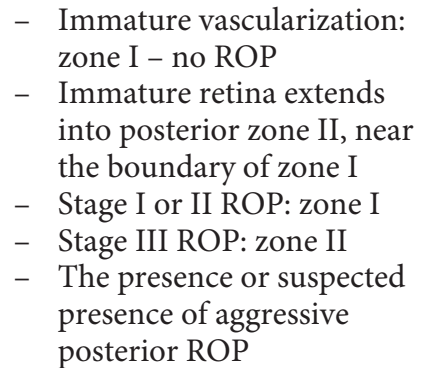 & $\begin{array}{l}\text { - } \text { Immature vascularization: } \\
\text { posterior zone II } \\
\text { - } \text { Stage 2 ROP: zone II } \\
\text { - Unequivocally regressing } \\
\quad \text { ROP: zone I }\end{array}$ & $\begin{array}{l}\text { - } \quad \text { Stage I ROP: zone II } \\
\text { - } \quad \text { Immature vascularization: } \\
\text { zone II - no ROP } \\
\text { - Unequivocally regressing } \\
\text { ROP: zone II }\end{array}$ & $\begin{array}{ll}\text { - } & \text { Stage I or II ROP: } \\
& \text { zone III } \\
-\quad & \text { Regressing ROP: } \\
& \text { zone III }\end{array}$ \\
\hline
\end{tabular}

ROP, retinopathy of prematurity.

$>31$ weeks or a birth weight $>1,500 \mathrm{~g}$ were also included if they were exposed to oxygen therapy for more than 7 days. Also, infants who were born between 31 and 34 weeks' gestational age were examined if they had a course of instability (such as sepsis, asphyxia, or ventilation). Moreover, older and heavier infants with an unstable clinical course, who were believed to be at high risk for ROP by their neonatologist, were also recruited for the study. The collected data included the birth weight, gender, gestational age and age at screening examination, and the findings of consecutive binocular indirect ophthalmoscopy examination.

The schedule for the timing of initial screening examination and follow-up examinations, and the time to stop screening examinations were determined according to the recommendations of the American Academy of Pediatrics, American Academy of Ophthalmology, and American Association for Pediatric Ophthalmology and Strabismus. The schedule is shown in Table 1.

Screening was performed by an experienced ophthalmologist. Each infant underwent a binocular indirect ophthalmoscopic examination for each eye with a 28D condensing lens and scleral depression following a pupillary dilation, which was achieved with $2.5 \%$ phenylephrine and $0.5 \%$ tropicamide. The examination was performed after instillation of topical anesthetic proparacaine and application of an eyelid speculum.

The screener carefully watched the eyes which were reaching the pre-threshold severity. The eyes were scheduled for treatment within $24-48 \mathrm{~h}$ of finding indicative signs. The treatment was given only if the ROP progressed to threshold severity (Type 1 ROP). Type 1 is defined as either development of stage III in zone I, any stage of ROP in zone I with plus disease, or development of stage II or III in zone II plus disease which is characterized by posterior tortuosity and dilatation of retinal blood vessels with decreased branching angels. Table 2 shows the characteristics of the treated cases including gestational age, body weight at birth, the stage and zone of the ROP, and time of initial and complete regression.

A total of 74 eyes of 37 preterm babies underwent intravitreal ranibizumab for ROP type 1 :

- 32 preterm babies were with plus disease stage III in zone II in both eyes ( 5 babies, 10 eyes were with the disease in posterior zone II).
- 2 preterm babies were with aggressive posterior ROP in both eyes.

- 3 preterm babies were with plus disease stage III in zone I. Intravitreal injections were performed under topical anesthesia after installation of povidone iodine. The lid specula were inserted, and injections were performed at $1.5 \mathrm{~mm}$ from the limbus with a 30 -gauge needle. The dose used was $0.3 \mathrm{mg} / 0.03 \mathrm{~mL}$ ranibizumab. Intraocular pressure and fundoscopic examination were performed after all injections. Infants were followed on day 1, and then after 1 week. All patients were followed weekly till the retina was completely vascularized. After that, they were evaluated every 3-4 months till the end of the study period.

\section{Results}

All eyes showed substantial response to intravitreal ranibizumab injection since day 1 . Regression of plus disease was observed from the following day which was followed by the regression of stage III. Figure 1 depicts a fundus photograph of one eye indicating the regression of ROP threshold disease 1 week after the treatment (panel d), while panels a-c demonstrate the rapid progression in only 2 weeks from ROP stage I, stage II, and stage III, respectively.

A total of 37 infants were included in the study. Mean gestational age \pm SD was $25.0 \pm 1.4$ weeks, median (range) was 25 weeks (23-28), with 51\% males. Mean birth weight $\pm \mathrm{SD}$ was $770.3 \pm 335.5 \mathrm{~g}$, median (range) was $720 \mathrm{~g}$ (510$2550)$. Mean post-conceptual age at injection \pm SD was $37.6 \pm 3.4$ weeks, median (range) was 37.0 weeks (32-49).

A total of 30 infants had initial regression $(+)$ within $24 \mathrm{~h}$ of treatment $(81.1 \%)$. Males had a higher rate of initial regression ( 89.5 vs. $72.2 \%$ for females); however, this result was not statistically significant $(p=0.18)$. Gesta- 
Table 2. Characteristics of the treated cases according to gestational age (GA in weeks), body weight at birth (BW in g), the stage and zone of the retinopathy of prematurity (ROP), and time of initial and complete regression

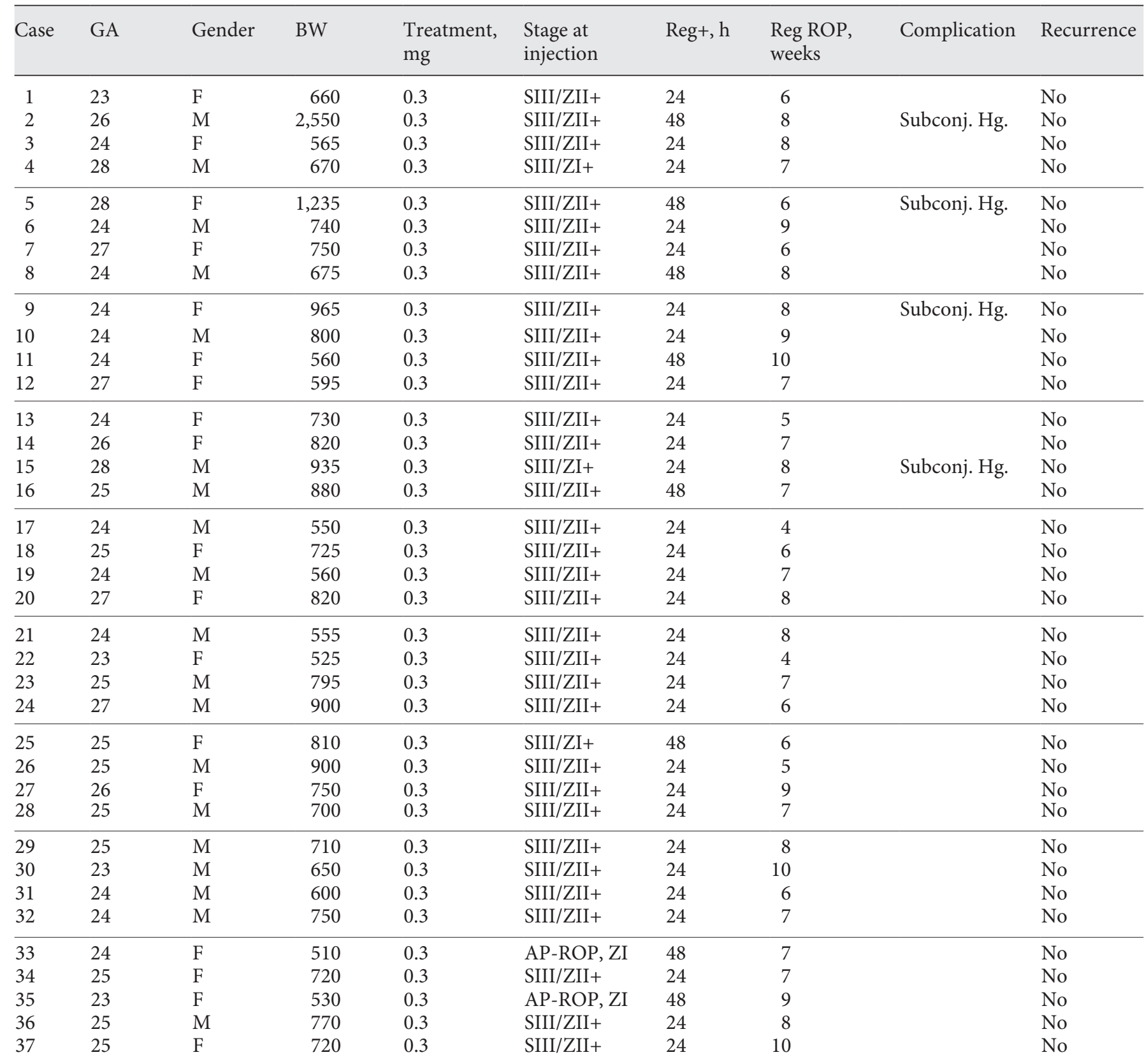

Reg+, initial regression of plus disease; Reg ROP, advancement of normal retinal vasculature; Subconj. Hg., subconjunctival hemorrhage.

tional age, birth weight, or conceptual age at injection were not related to regression (+) within $24 \mathrm{~h}$.

All 37 patients had regression of ROP within 10 weeks of injection, 21 patients (56.8\%) had regression of ROP within 7 weeks of injection. Gestational age, birth weight, gender, or conceptual age at injection were also not related to earlier regression of ROP (within 7 weeks).

All of the cases had completely regressed tortuosity and dilatation of retinal blood vessels by the end of the 
Fig. 1. Fundus photograph for a single preterm baby (gestational age at birth $=32$ weeks, body weight $=510 \mathrm{~g}$ ). a During the first week (week 32), indicating ROP stage $\mathrm{I}$ in zone I/posterior zone II; $\mathbf{b}$ during the second week (week 33), indicating progression of ROP to stage II; c during the third week (week 34), indicating progression of ROP to stage 3 at zone I and posterior zone II; d during the fourth week (week 35) and one week after the injection, showing regression of ROP threshold disease.
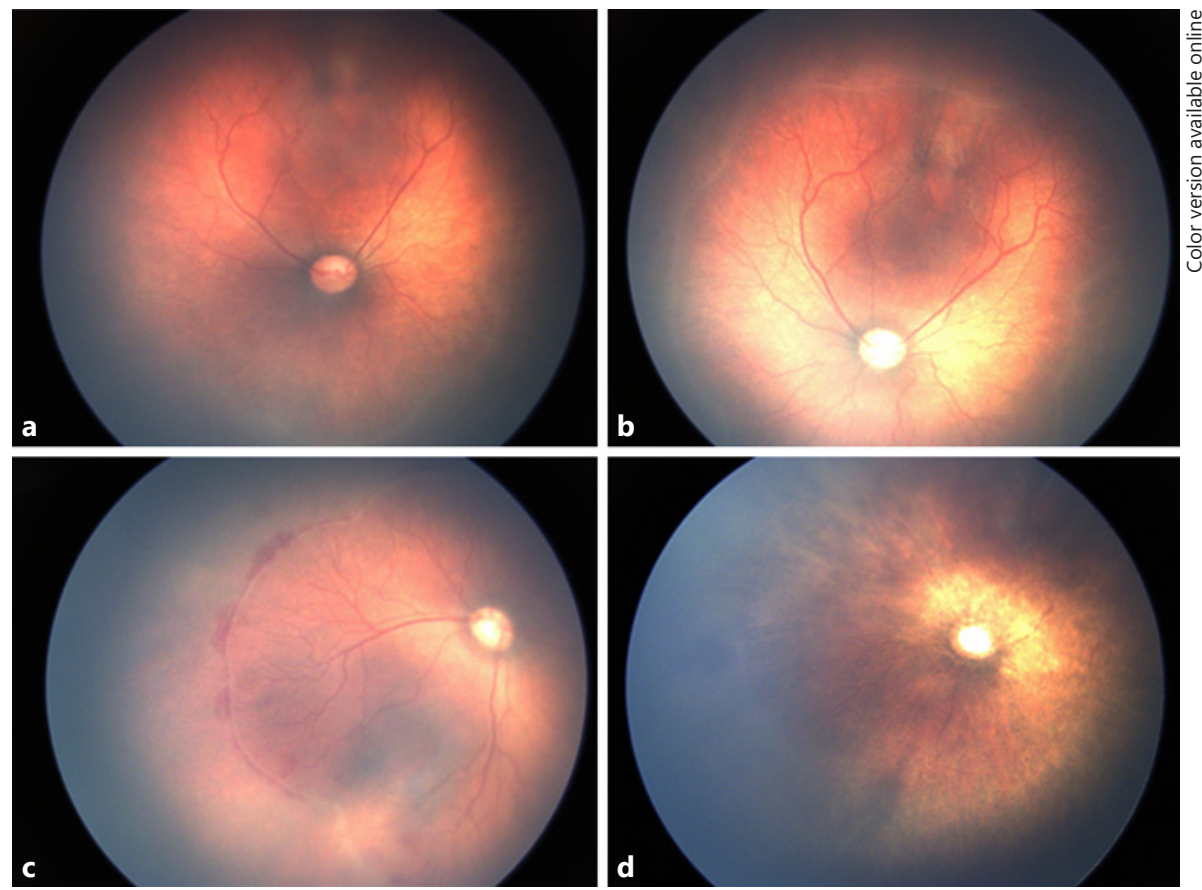

first week with a decreasing volume of ridge. There was a total regression of stage III after 4-8 weeks. The vessels started growing over the ridge towards the periphery, and this was complete in most infants in 3 months. The close follow-up period was 12 months in 17 infants and 6 months in the rest of the treatment group.

With regard to adverse effects, only 4 of our patients developed subconjunctival hemorrhage in one of their eyes. Despite that almost all premature babies had some degree of intraventricular hemorrhage prior to the intravitreal injection, none of them had an increase in the degree of intraventricular hemorrhage after the injection. None of the treated eyes had a post-injection elevation of intraocular pressure that required intervention. Regarding neurodevelopmental issues, 5 of our patients are still being followed in the neurosurgery clinic and the rehabilitation department, as they underwent ventriculo-peritoneal shunt surgeries for hemorrhagic hydrocephalus. We believe that this presentation is not associated with the treatment, and it is a known consequence of prematurity. One 4-year-old is scheduled for squint surgery, and the other four are regular patients in the optometry clinic being followed up for amblyopia therapy for anisometropia. No other systemic side effects were noted both during the treatment and in the follow-up period.

\section{Discussion}

Our results are consistent with the studies of Menke et al. [16] and Castellanos et al. [17] who also reported complete resolution of stage III ROP with plus disease in zone II with no recurrence during the 6-month follow-up period. They used the same dose and the mean gestational age as well; the mean postmenstrual age at injection was also not statistically different from that of our patients. However, these two studies had small sample sizes of 6 eyes each. Chen et al. [18] compared the efficacy of intravitreal ranibizumab with bevacizumab in two groups of premature children with ROP. The authors concluded similar efficacy of both drugs in terms of regression of the disease. Another retrospective study conducted by Arámbulo et al. [19] in Brazil compared the results obtained with intravitreal ranibizumab treatment alone and combined treatment with ranibizumab and laser photocoagulation. $87.5 \%$ of the cases showed favorable results in terms of regression of neovascularization with ranibizumab $(0.25 \mathrm{mg})$ in 16 eyes, while $12.5 \%$ of the patients had unfavorable results in the form of disease progression to stage 4 and 5 . In the group treated with ranibizumab and laser, favorable results were only achieved in $70.7 \%$ of the eyes.

In our study, we had 3 extremely premature infants with a very low gestational age of 23 weeks and birth 
weight ranging between 530 and $660 \mathrm{~g}$. They responded very well to the treatment. This is in agreement with a case of extremely low birth weight ( $480 \mathrm{~g}$ with a gestational age of 23 weeks) with Rush disease reported by Lin et al. [20]. The infant was first treated with combined intravitreal bevacizumab and laser therapy, and no progress was achieved. After a single injection of intravitreal ranibizumab, complete resolution of the disease was achieved with no recurrence during a follow-up period of 2 years.

It is worth mentioning that fluorescein angiography is a more sensitive tool for the detection of peripheral vascular growth compared to fundoscopic examination, the clinical tool used in our study. However, diagnosis of recurrence was confirmed in many studies based on clinical examination alone [21-25].

Our results are consistent with the reports mentioned above regarding the initial response and recurrence; however, we cannot directly compare the results as there is a difference in sample size as well as the anti-VEGF agent and dose used. The dose of ranibizumab used in our patients was $0.3 \mathrm{mg}$, whereas in all of the mentioned reports (except for the one by Menke et al. [16]) the dose used was $0.2-0.25 \mathrm{mg}$. Despite the relatively smaller dose, the above-mentioned reports have shown almost $100 \%$ positive outcomes in terms of initial regression.

Several reports indicated late recurrence of ROP after different anti-VEGF treatment [26-28]. A randomized clinical trial concluded that single-dose anti-VEGF monotherapy is not recommended for ROP in zone II due to a high probability of early recurrence [29]. They reported that 26 eyes out of 50 (52\%) had a recurrence of ROP within a maximum follow-up period of 79 weeks after intravitreal injection of ranibizumab. A similar observation was reported by Mintz-Hittner et al. [13] for bevacizumab monotherapy. Other studies reported a higher incidence of recurrence in patients treated with intravitreal ranibizumab compared to bevacizumab-treated cases [21-25]. The ranibizumab dose used in those studies was between 0.2 and $0.25 \mathrm{mg}$, while in our study, a dose of $0.3 \mathrm{mg}$ was used. This may explain the absence of recurrence in our study. The lower postconceptional age at the time of treatment, the zone, and extent of disease in the affected eye are possible determinant factors for recurrence. Some reports showed a reduced risk of recurrence with ROP in zone I after treatment with anti-VEGF and bevacizumab [13, 30].

This study showed lower adverse effects compared to many studies including the study of Menke et al. [16] who reported that paracentesis had to be performed in

3 out of the 6 eyes included in their study. Other studies demonstrated a higher incidence of neurodevelopmental disabilities, starting from 18 months of corrected gestational age, in patients treated with bevacizumab [31, 32].

\section{Conclusion}

We conclude that the use of a single intravitreal injection of $0.3 \mathrm{mg}$ ranibizumab is effective in treating advanced ROP stage III mainly in zone II and also in zone I. The treatment led to complete regression of plus disease within $24-48 \mathrm{~h}$. The retinal vessels progressed anteriorly, and complete retinal vascularization was achieved within 4-10 weeks of treatment. Although treatment with intravitreal ranibizumab is generally well tolerated, long-term ocular and systemic effects need to be investigated with further prospective trials using larger sample sizes and longer follow-up periods. More studies are needed to optimize the dose of ranibizumab and other anti-VEGF agents.

\section{Statement of Ethics}

This study was approved by the Institutional Review Board of King Abdullah International Medical Research Center, and both verbal and written informed consents were obtained from the parents of all patients.

\section{Disclosure Statement}

All authors have no conflict of interest to disclose.

References

Med Princ Pract 2019;28:526-532 DOI: $10.1159 / 000500310$
1 Hellström A, Smith LE, Dammann O. Retinopathy of prematurity. Lancet. 2013 Oct; 382(9902): 1445-57.

2 Liu L, Tian T, Zheng CX, Ileana V, Ioana A, Tatiana C, et al. Risk factors and laser therapy for retinopathy of prematurity in neonatal intensive care unit. World J Pediatr. 2009 Nov; 5(4):304-7.

3 Lee J, Dammann O. Perinatal infection, inflammation, and retinopathy of prematurity. Semin Fetal Neonatal Med. 2012 Feb;17(1): 26-9.

4 Shah PK, Prabhu V, Karandikar SS, Ranjan R, Narendran V, Kalpana N. Retinopathy of prematurity: Past, present and future. World J Clin Pediatr. 2016 Feb;5(1):35-46.

5 Cross KW. Cost of preventing retrolental fibroplasia? Lancet. 1973 Oct;2(7835):954-6. 
6 Blencowe H, Lawn JE, Vazquez T, Fielder A, Gilbert C. Preterm-associated visual impairment and estimates of retinopathy of prematurity at regional and global levels for 2010 . Pediatr Res. 2013 Dec;74(S1 Suppl 1):35-49.

7 Fierson WMAmerican Academy of Pediatrics Section on OphthalmologyAmerican Academy of OphthalmologyAmerican Association for Pediatric Ophthalmology and Strabismus; American Association of Certified Orthoptists. Screening examination of premature infants for retinopathy of prematurity. Pediatrics. 2013 Jan;131(1):189-95.

8 Lad EM, Nguyen TC, Morton JM, Moshfeghi DM. Retinopathy of prematurity in the United States. Br J Ophthalmol. 2008 Mar;92(3): $320-5$.

9 Wilkinson AR, Haines L, Head K, Fielder AR. UK retinopathy of prematurity guideline. Early Hum Dev. 2008 Feb;84(2):71-4.

10 Smith LE. Through the eyes of a child: understanding retinopathy through ROP the Friedenwald lecture. Invest Ophthalmol Vis Sci. 2008 Dec;49(12):5177-82.

11 Zhao M, Xie WK, Bai YJ, Huang LZ, Wang B, Liang $\mathrm{JH}$, et al. Expression of total vascular endothelial growth factor and the anti-angiogenic VEGF165b isoform in the vitreous of patients with retinopathy of prematurity. Chin Med J (Engl). 2015 Sep;128(18):2505-9.

12 Early Treatment For Retinopathy Of Prematurity Cooperative Group. Revised indications for the treatment of retinopathy of prematurity: results of early treatment for retinopathy of prematurity randomized trial. Arch Ophthalmol. 2003 Dec;121(12):168494.

13 Mintz-Hittner HA, Kennedy KA, Chuang AZ; BEAT-ROP Cooperative Group. Efficacy of intravitreal bevacizumab for stage $3+$ retinopathy of prematurity. N Engl J Med. 2011 Feb;364(7):603-15.

14 Leskov I, Mukai S. Laser therapy versus antiVEGF agents for treatment of retinopathy of prematurity. Int Ophthalmol Clin. 2015 Fall; 55(4):81-90.
15 Zhou Y, Jiang Y, Bai Y, Wen J, Chen L. Vascular endothelial growth factor plasma levels before and after treatment of retinopathy of prematurity with ranibizumab. Graefes Arch Clin Exp Ophthalmol. 2016 Jan;254(1):31-6.

16 Menke MN, Framme C, Nelle M, Berger MR, Sturm V, Wolf S. Intravitreal ranibizumab monotherapy to treat retinopathy of prematurity zone II, stage 3 with plus disease. BMC Ophthalmol. 2015 Mar;15(1):20.

17 Castellanos MA, Schwartz S, García-Aguirre G, Quiroz-Mercado H. Short-term outcome after intravitreal ranibizumab injections for the treatment of retinopathy of prematurity. Br J Ophthalmol. 2013 Jul;97(7):816-9.

18 Chen SN, Lian I, Hwang YC, Chen YH, Chang $\mathrm{YC}$, Lee $\mathrm{KH}$, et al. Intravitreal anti-vascular endothelial growth factor treatment for retinopathy of prematurity: comparison between Ranibizumab and Bevacizumab. Retina. 2015 Apr;35(4):667-74.

19 Arámbulo O, Dib G, Iturralde J, Duran F, Brito M, Fortes Filho JB. Intravitreal ranibizum$\mathrm{ab}$ as a primary or a combined treatment for severe retinopathy of prematurity. Clin Ophthalmol. 2015 Oct;9:2027-32.

20 Lin CJ, Chen SN, Hwang JF. Intravitreal ranibizumab as salvage therapy in an extremely low-birth-weight infant with rush type retinopathy of prematurity. Oman J Ophthalmol. 2012 Sep;5(3):184-6.

21 Wong RK, Hubschman S, Tsui I. Reactivation of retinopathy of prematurity after ranibizumab treatment. Retina. 2015 Apr;35(4): 675-80.

22 Erol MK, Coban DT, Sari ES, Bilgin AB, Dogan B, Ozdemir O, et al. Comparison of intravitreal ranibizumab and bevacizumab treatment for retinopathy of prematurity. Arq Bras Oftalmol. 2015 Nov-Dec;78(6):340-3.

23 Chan JJ, Lam CP, Kwok MK, Wong RL, Lee GK, Lau WW, et al. Risk of recurrence of retinopathy of prematurity after initial intravitreal ranibizumab therapy. Sci Rep. 2016 Jun; 6(1):27082.

24 Yi Z, Su Y, Zhou Y, Zheng H, Ye M, Xu Y, et al. Effects of intravitreal ranibizumab in the treatment of retinopathy of prematurity in Chinese infants. Curr Eye Res. 2016 Aug; 41(8):1092-7.
25 Baumal CR, Goldberg RA, Fein JG. Primary intravitreal ranibizumab for high-risk retinopathy of prematurity. Ophthalmic Surg Lasers Imaging Retina. 2015 Apr;46(4):432-8.

26 Snyder LL, Garcia-Gonzalez JM, Shapiro MJ, Blair MP. Very late reactivation of retinopathy of prematurity after monotherapy with intravitreal bevacizumab. Ophthalmic Surg Lasers Imaging Retina. 2016 Mar;47(3):2803.

$27 \mathrm{Hu}$ J, Blair MP, Shapiro MJ, Lichtenstein SJ, Galasso JM, Kapur R; Hu J1. Blair MP, Shapiro MJ, Lichtenstein SJ, Galasso JM, Kapur R. Reactivation of retinopathy of prematurity after bevacizumab injection. Arch Ophthalmol. 2012;130(8):1000-6.

28 Hajrasouliha AR, Garcia-Gonzales JM, Shapiro MJ, Yoon H, Blair MP. Reactivation of Retinopathy of Prematurity Three Years After Treatment With Bevacizumab. Ophthalmic Surg Lasers Imaging Retina. 2017 Mar;48(3): 255-9.

29 Zhang G, Yang M, Zeng J, Vakros G, Su K, Chen $M$, et al. Comparison of intravitreal injection of ranibizumab versus laser therapy for zone II treatment-requiring retinopathy of prematurity. Retina. 2017 Apr;37(4):7107.

30 Reynolds JD. Bevacizumab for retinopathy of prematurity. N Engl J Med. 2011 Feb;364(7): 677-8.

31 Morin J, Luu TM, Superstein R, Ospina LH, Lefebvre F, Simard MN, et al.; Canadian Neonatal Network and the Canadian Neonatal Follow-Up Network Investigators. Neurodevelopmental outcomes following bevacizum$\mathrm{ab}$ injections for retinopathy of prematurity. Pediatrics. 2016 Apr;137(4):e20153218.

32 Araz-Ersan B, Kir N, Tuncer S, AydinogluCandan O, Yildiz-Inec D, Akdogan B, et al. Preliminary anatomical and neurodevelopmental outcomes of intravitreal bevacizumab as adjunctive treatment for retinopathy of prematurity. Curr Eye Res. 2015 May;40(6): 585-91. 